\title{
CONCEPCIONES DE ESTUDIANTES UNIVERSITARIOS SOBRE APRENDIZAJE COLABORATIVO APOYADO EN TECNOLOGÍAS DIGITALES
}

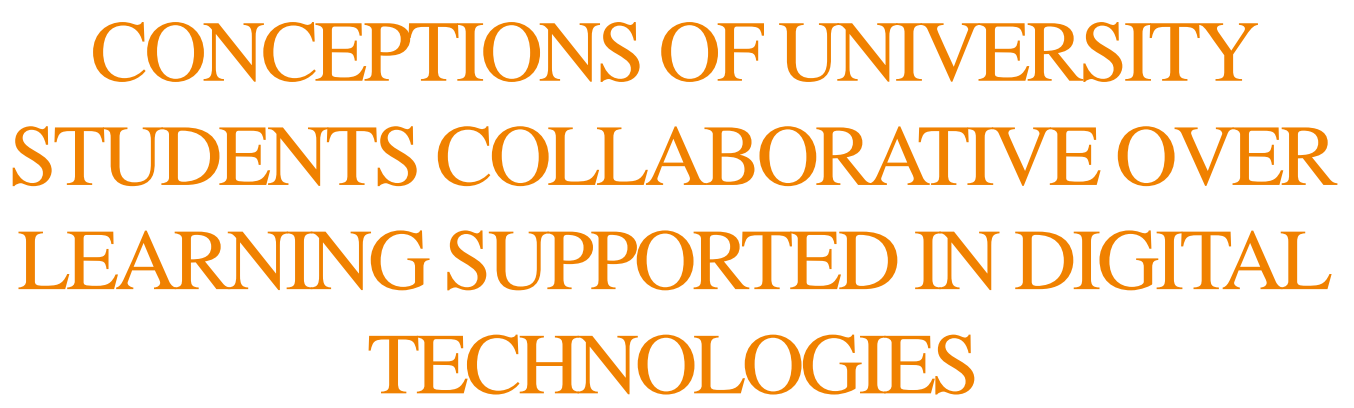

Audin Aloiso Gamboa Suarez ${ }^{2}$

Raúl Prada Núñez ${ }^{3}$

William Rodrigo Avendaño Castro ${ }^{4}$

\section{RESUMEN}

Las tecnologías digitales requieren de una mirada innovadora que amplíe su accionar

$1 \quad$ Proyecto de investigación Avalado por el Grupo de Investigación GIESPPAZ y GIPPEP y apoyado por los semilleros de investigación en Educación Matemática y SIPRILA de la Universidad Francisco de Paula Santander 2 Doctor en Ciencias de la Educación por la Universidad de Cartagena. Docente investigador de la Universidad Francisco de Paula Santander. E-mail: audingamboa@ufps. edu.co. Orcid: 0000-0001-9755-6408

3 Magister en Educación Matemática. Facultad de Educación, Artes y Humanidades. E-mail: raulprada@ufps. edu.co. Orcid: 0000-0001-6145-1786

4 Doctor en Ciencias Sociales y Humanas. E-mail: williamavendano@ufps.edu.co, Orcid: 0000-0002-75108222 hacia una interacción social y que estas se adapten a los cambios en los nuevos roles de la educación que demanda un aprendizaje colaborativo. El presente texto muestra los resultados de un estudio que buscó identificar las concepciones que tienen estudiantes universitarios sobre el aprendizaje colaborativo apoyado en tecnologías digitales. El paradigma de investigación se enmarca en el enfoque cuantitativo - descriptivo transversal siguiendo un diseño de campo, con una muestra de 200 estudiantes elegida de forma no probabilística. Los resultados demuestran que con el desarrollo 
de las actividades grupales, los estudiantes potenciaron sus competencias tecnológicas haciéndolos gestores de su propia cualificación gracias a la interacción con sus compañeros de trabajo y al reconocimiento de la aplicación de sus competencias en la solución de problemas cotidianos.

\section{PALABRAS CLAVE}

Aprendizaje colaborativo, educación superior, tecnologías digitales, virtualidad

\section{ABSTRACT}

Digital technologies require an innovative look that broadens their actions towards social interaction and that these adapt to the changes in the new roles of education that collaborative learning demands. This text shows the results of a study that sought to identify the conceptions that university students have about collaborative learning supported by digital technologies. The research paradigm is framed in the crosssectional quantitative-descriptive approach following a field design, with a sample of 200 students chosen in a non-probabilistic way. The results show that with the development of group activities, students enhanced their technological skills, making them managers of their own qualifications thanks to the interaction with their co-workers and the recognition of the application of their skills in solving everyday problems.

\section{KEYWORDS}

Collaborative learning, higher education, digital technologies, virtuality

\section{INTRODUCCIÓN}

Los cambios que se han generado en la actualidad dan relevancia al aprendizaje para el desarrollo social, pero también ponen en duda la capacidad institucional para cumplir con sus objetivos y funciones a nivel mundial, partiendo de problemas en el ordenamiento y funcionamiento dentro de la educación, donde aspectos como la participación de alumnos y equipo docente en actividades de aula, el proyecto educativo y las directrices curriculares (Coll, 2014), pueden verse incluidas dentro del tema de aprendizaje colaborativo de que se discernirá a través del presente documento.

Los mencionados cambios sociales configurados son una suma de hechos históricos como la Revolución Francesa, que han dado otro sentido a la escuela, haciéndola "[...]pasar metodológicamente hablando de la estructura clásica de la formación libresca, individual y bancaria, a un proceso de formación más abierto, grupal social y dinámico" (Rubia y Guitert, 2014, p. 10), donde la tecnología empezó a fortalecerse en el ámbito colaborativo a partir de los años 90 con motivo de encontrar una concepción común de los problemas mediante la coordinación, lo sincrónico y el trabajo en equipo. Además, los autores citados mencionan que si aquel ideal objetivo de la educación es una sociedad sinérgica y modélica, esta deberá promover la igualdad mediante la comunicación y el acuerdo de comportamientos de interrelación. Así, se proponen 4 aspectos básicos del aprendizaje colaborativo:

[...]la necesaria situación que permita la colaboración entre personas del mismo estatus (un docente y su alumnado, por ejemplo); las interacciones que han de facilitar la colaboración, por ejemplo cuando se produce negociación en vez de instrucción; los propios mecanismos de aprendizaje, apoyados en dinámicas de asimilación y acomodación, por ejemplo, desde la perspectiva del acuerdo grupal sobre lo aprendido; y por último, los efectos de aprendizaje colaborativo que se apoya en distintas dinámicas de registro de la acción de aprender y que construyen resultados más allá de los finales del dominio del 
contenido que se aprende (Dillenbourg, citado en Rubia y Guitert, 2014).

Parece ser que para Hernández et al (2014), la clave para entender lo que es el aprendizaje colaborativo, son los intercambios y el trabajo en comunidad tras la persecución de unos fines de aprendizaje, aspectos que se pueden ver favorecidos por la planificación, que, dentro del ámbito virtual, debe contemplar recursos tecnológicos, una metodología y la estructuración de los grupos de trabajo, además de la redacción de acuerdos en el grupo. Por otro lado, estos autores afirman que el aprendizaje colaborativo debe articular las competencias que se desarrollarán dentro del programa como objetivo del aprendizaje al método colaborativo que se usará y este a las actividades de aprendizaje postuladas en los objetivos; además, se menciona la selección del método y tipología que sirva para la resolución de problemas complejos que requieren respuestas complejas - variación de acciones y decisiones -; los recursos de comunicación con los estudiantes sobre el trabajo colaborativo, y las características y el proceso de formación del grupo (Hernández, 2014).

Autores como Calzadilla (2002), se dirigen hacia aquellas tareas complejas, que dan respuesta a problemas complejos desde el colectivo y atribuyen a la ponderación y el equilibrio la eficacia de la constitución de grupos felices a través de la percepción emocional, donde el aprendizaje colaborativo, como propiciador de la estructura cognoscitiva, se verá favorecido. Así, esta autora afirma que

Existe incidencia de la inteligencia emocional en el comportamiento de la labor del individuo en las organizaciones, y que ellas influyen de manera significativa en las relaciones interpersonales para crear un clima que active la creatividad y den apertura a escenarios de participación en la toma de decisiones que impulsen la mejor gestión como producto de un trabajo en equipo sinérgico (Calzadilla, 2002, p. 3).

Así las cosas, las herramientas digitales requieren de una mirada innovadora que amplíe su accionar hacia una interacción social en ella que se adapten a los cambios en roles de la educación que demanda un aprendizaje colaborativo, lo que hace visible la necesidad de capacitación en el manejo de estas (Gonzáles, 2006; Hernández, Arévalo y Gamboa, 2016; Prada, Hernández y Gamboa, 2019). En este sentido, Fernández et al (1999), ve la necesidad de definir tecnologías softwares como las "[...]provenientes del área de Computer Supported Cooperative Work (CSCW), para organizar y definir recursos, espacios y actividades compartidas por grupos de individuos" (p. 14), el cual puede ser visto a su vez como un enfoque educativo, junto con el Groupware, que se refiere al trabajo en grupo, donde las personas colaboran entre ellas mediante redes de computadoras, aumentando la eficacia comunicativa, de coordinación y de cooperación. El recién citado autor presenta además la clasificación espacio - temporal de estas aplicaciones de Johansen, a saber: "mismo tiempo - mismo lugar", "mismo tiempo - diferente lugar:", "diferente tiempo - mismo lugar:", "diferente tiempo - diferente lugar:" ( $p$. 15). Finalmente, este autor concluye que:

Los sistemas actuales ofrecen, en mayor o menor medida: (1) Acceso/ Intercambio de material a través de libros electrónicos que muestran por pantalla la información a la que se accede secuencialmente $\mathrm{o}$ con hipertextos muy sencillos; (2) comunicación profesor/alumno para el intercambio de preguntas/respuestas e intercambio de material; (3) hipertextos complejos que intentan organizan la información según las necesidades del alumno; y 
(4) trabajo en grupo en sistemas que permiten realizar tareas entre varios alumnos con o sin ayuda del profesor, la comunicación síncrona y/o asíncrona de todo el grupo, sistemas de ayuda más sofisticados y gestión de las tareas del grupo (p. 20).

Lo anterior refleja cómo el trabajo colaborativo se presenta en diversas formas tales como la transmisión y recepción de información compartida por estos medios de forma práctica; el intercambio de percepciones, nociones y experiencias, además de material académico; organización de las necesidades grupales y, tareas y trabajos conjuntos. Entonces, hablar de aprendizaje colaborativo en contextos virtuales, tiene ciertos retos, que, si bien implican una ardua tarea para los diseños de aplicativos y para quienes los aplican, también pueden facilitar una nueva forma de interacción, donde estudiantes y docentes se sientan cómodos y logren articularse en ideas, percepciones y recursos, en un esfuerzo por llevar su práctica académica hacia las necesidades de la actualidad y como contingencia y practicidad en las redes sociales construidas tras interacciones productivas.

\section{METODOLOGÍA}

Esta investigación se ajusta a las características del enfoque cuantitativo a nivel descriptivo transversal siguiendo un diseño de campo, puesto que los datos son recolectados de una muestra elegida no probabilísticamente (muestreo voluntario) de estudiantes de la Facultad de Educación de una universidad pública del nororiente colombiano. Se les invitó a diligenciar un formulario en línea, luego los datos recolectados se procesaron con el fin analizar la validez de la escala utilizada dado que fue un instrumento Ad hoc, para posteriormente procesarlos de forma descriptiva determinando porcentajes y frecuencias en cada ítem sobre las diversas opciones de respuesta.
Como ya se mencionó se diseñó un cuestionario compuesto principalmente por tres secciones: inicia con una breve descripción demográfica del estudiante, seguida de veintitrés reactivos los cuales son evaluados mediante una escala Likert de frecuencia a cinco niveles con la característica de tener dos niveles de percepción positiva (Siempre y Casi siempre), un nivel intermedio (Algunas veces) y dos niveles de percepción negativa (Casi nunca y Nunca).

Durante la ventana de recolección de datos se logró conformar una muestra de 200 estudiantes, los cuales recibieron la invitación a diligenciar el instrumento por medio del director de programa académico quien compartió el link desde la cuenta institucional de su programa. Finalizado el tiempo de recolección se descarga del Google Form el archivo de Excel con los datos, para luego ser exportados al SPSS v25, software con el que se realiza el procesamiento de los datos.

\section{RESULTADOS}

Como ya mencionó en la sección anterior, el instrumento se compone de tres secciones por lo cual los resultados serán presentados en ese orden.

\section{Primera Sección: Perfil del informante}

Respecto a las características del grupo de informantes, se destaca que el $65 \%$ tienen edades que oscilan entre los 18 y 20 años, con predominio del género femenino que resulta coherente dado que están matriculados en los programas de Licenciatura en Educación Infantil y Trabajo Social, cuyos estudiantes son en su mayoría mujeres. Respecto al semestre académico que cursan actualmente, se pudo identificar que aproximadamente el $78.5 \%$ de ellos están matriculados entre el tercero y el quinto semestre. 
Tabla 1. Perfil demográfico y académico del informante

\begin{tabular}{|c|c|c|c|}
\hline Variable & Niveles de respuesta & Frecuencia & Porcentaje \\
\hline \multirow{4}{*}{ Edad } & Entre 18 y 20 años & 131 & $65.5 \%$ \\
\hline & Entre 21 y 23 años & 64 & $32.0 \%$ \\
\hline & Entre 24 y 26 años & 5 & $2.5 \%$ \\
\hline & Total & 200 & $100.0 \%$ \\
\hline \multirow{3}{*}{ Género } & Femenino & 176 & $88.0 \%$ \\
\hline & Masculino & 24 & $12.0 \%$ \\
\hline & Total & 200 & $100.0 \%$ \\
\hline \multirow{5}{*}{$\begin{array}{c}\text { Programa académico } \\
\text { que cursa }\end{array}$} & $\begin{array}{c}\text { Licenciatura en Ciencias Naturales y } \\
\text { Educación Ambiental }\end{array}$ & 27 & $13.5 \%$ \\
\hline & Licenciatura en Matemáticas & 29 & $14.5 \%$ \\
\hline & Licenciatura en Educación Infantil & 87 & $43.5 \%$ \\
\hline & Trabajo Social & 57 & $28.5 \%$ \\
\hline & Total & 200 & $100.0 \%$ \\
\hline \multirow{7}{*}{$\begin{array}{l}\text { Semestre académico } \\
\text { que cursa actualmente }\end{array}$} & Tercero & 73 & $36.5 \%$ \\
\hline & Cuarto & 65 & $32.5 \%$ \\
\hline & Quinto & 19 & $9.5 \%$ \\
\hline & Sexto & 23 & $11.5 \%$ \\
\hline & Séptimo & 10 & $5.0 \%$ \\
\hline & Octavo & 10 & $5.0 \%$ \\
\hline & Total & 200 & $100.0 \%$ \\
\hline
\end{tabular}

\section{Segunda Sección: Percepción del trabajo mediado por TIC.}

Es importante señalar que la fiabilidad de una escala corresponde a una medida que permite evaluar si el instrumento utilizado mide el constructo para el que fue diseñado y si esta medición es estable en el tiempo. Para tal fin se recurre a determinar el estadístico Alfa de Cronbach utilizando el software SPSS, que reporta un valor de 0.896 para la escala con 23 reactivos evaluados utilizando una escala Likert con cinco niveles. Este valor es ligeramente inferior a 0.90 , valor que según Celina Oviedo y Campo Arias (2005) sería el máximo admisible, y en caso de que fuese mayor, se interpretaría como que existe evidencia de la presencia de ítems redundantes, es decir, que algunos reactivos que están midiendo la misma característica del constructo.
Verificada la pertinencia del instrumento utilizado, se puede avanzar al análisis descriptivo del instrumento, para lo cual los dos niveles de valoración positiva (calificaciones 5 y 4) se han agrupado en una nueva categoría que se denomina Siempre, el nivel intermedio se mantiene igual, y los dos niveles de valoración negativa (puntuaciones 1 y 2) se agrupan en la categoría Nunca. Este proceso se realiza con el fin de simplificar la interpretación de los resultados a la luz de que todos los reactivos han sido redactados de forma afirmativa. 


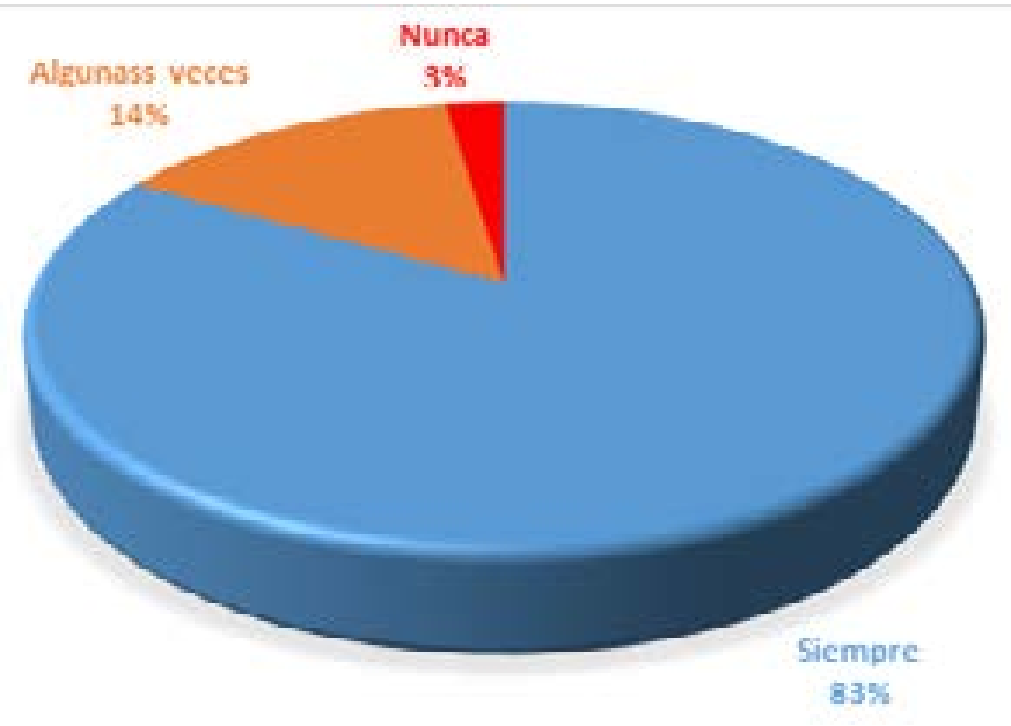

Gráfico 1. Promedio general sobre el uso de actividades de trabajo colaborativo en época de no presencialidad

El gráfico 1 permite evidenciar que en opinión del $83 \%$ de los estudiantes encuestados existe una percepción favorable sobre el uso adecuado e implementación de actividades que potenciaron el trabajo colaborativo durante el desarrollo pedagógico de actividades académicas asistidas con recursos tecnológicos.

Por medio de la Tabla 2 se realiza una revisión pormenorizada de los diversos aspectos que se indagaron en los estudiantes informantes. Los estudiantes reconocen varias fortalezas en las actividades promocionadas por los docentes durante este tiempo de actividades no presenciales, dentro de las que destacan:

- El material suministrado por los docentes definía metas claras, con instrucciones precisas, con actividades pertinentes y tiempos coherentes.

- La organización de las actividades por medio de grupos de trabajo fundamentados en el trabajo colaborativo permitió a los estudiantes acercarse a sus compañeros a pesar de la no presencialidad, generando espacios en donde se organizaban roles, se definían tareas, se asumían responsabilidades y todos aportaban a un objetivo común, logrando la entrega de actividades en dónde todos fueron constructores del mismo.

- Con el desarrollo de estas actividades los estudiantes potenciaron sus competencias tecnológicas haciéndolos gestores de su propia cualificación gracias a la interacción con sus compañeros de trabajo y al reconocimiento de la aplicación de sus competencias en la solución de problemas cotidianos.

- Finalmente, en cuanto al rol del docente, se evidenció que en opinión de los estudiantes evaluados, consideraron al docente un acompañante en el proceso que garantizó que se mantuvieran encaminados hacia los objetivos de aprendizaje, puesto que 
definió rúbricas claras, aclaró las dudas que surgieron en el proceso de forma adecuada y realizó una adecuada valoración de los aportes que realizaban los estudiantes en actividades como los foros de discusión.

Tabla 2. Descripción detallada de la valoración

\begin{tabular}{|c|c|c|c|}
\hline Ítem & Siempre & $\begin{array}{l}\text { Algunas } \\
\text { veces }\end{array}$ & Nunca \\
\hline $\begin{array}{l}\text { El material de consulta recomendado en las } \\
\text { asignaturas fue útil para el desarrollo de las } \\
\text { actividades planteadas. }\end{array}$ & $86.0 \%$ & $13.5 \%$ & $0.5 \%$ \\
\hline $\begin{array}{l}\text { Las actividades desarrolladas potenciaron la } \\
\text { interacción entre los miembros del grupo. }\end{array}$ & $83.5 \%$ & $15.0 \%$ & $1.5 \%$ \\
\hline $\begin{array}{l}\text { Los objetivos de las actividades a desarrollar } \\
\text { en el trabajo colaborativo se exponen de forma } \\
\text { clara y precisa. }\end{array}$ & $81.5 \%$ & $18.5 \%$ & $0.0 \%$ \\
\hline $\begin{array}{l}\text { El tiempo programado para el desarrollo del } \\
\text { trabajo colaborativo fue suficiente. }\end{array}$ & $76.5 \%$ & $21.5 \%$ & $2.0 \%$ \\
\hline $\begin{array}{l}\text { El equipo de trabajo conformado, le permitió } \\
\text { sentirse acompañado y partícipe de un grupo. }\end{array}$ & $85.0 \%$ & $15.0 \%$ & $0.0 \%$ \\
\hline $\begin{array}{l}\text { El grupo de trabajo colaborativo debe ser de } \\
\text { cinco estudiantes. }\end{array}$ & $73.5 \%$ & $29.0 \%$ & $7.5 \%$ \\
\hline $\begin{array}{l}\text { Usted hizo comentarios de los aportes de sus } \\
\text { compañeros en el foro. }\end{array}$ & $68.0 \%$ & $16.5 \%$ & $15.5 \%$ \\
\hline $\begin{array}{l}\text { Participó activamente en la construcción del } \\
\text { producto final del trabajo colaborativo. }\end{array}$ & $89.5 \%$ & $8.5 \%$ & $2.0 \%$ \\
\hline $\begin{array}{l}\text { Cada participante tenía un rol y una } \\
\text { responsabilidad en el desarrollo del trabajo } \\
\text { colaborativo. }\end{array}$ & $92.5 \%$ & $7.5 \%$ & $0.0 \%$ \\
\hline $\begin{array}{l}\text { Sus compañeros hicieron comentarios o } \\
\text { correcciones sobre sus aportes. }\end{array}$ & $64.0 \%$ & $20.5 \%$ & $15.5 \%$ \\
\hline $\begin{array}{l}\text { La metodología del trabajo colaborativo virtual } \\
\text { le permitió sentirse activo en la construcción de } \\
\text { su propio conocimiento. }\end{array}$ & $81.0 \%$ & $16.5 \%$ & $2.5 \%$ \\
\hline $\begin{array}{l}\text { Se siente más competente en el uso de las } \\
\text { herramientas informáticas luego de haber } \\
\text { terminado el proceso. }\end{array}$ & $81.0 \%$ & $18.0 \%$ & $1.0 \%$ \\
\hline $\begin{array}{l}\text { Es bueno para su formación ver distintas } \\
\text { alternativas de solución al abordar un problema. }\end{array}$ & $96.0 \%$ & $4.0 \%$ & $0.0 \%$ \\
\hline $\begin{array}{l}\text { Los temas tratados en los trabajos colaborativos } \\
\text { le son útiles en su vida profesional. }\end{array}$ & $94.0 \%$ & $6.0 \%$ & $0.0 \%$ \\
\hline
\end{tabular}




\begin{tabular}{llll}
\hline $\begin{array}{l}\text { Es de vital importancia la interacción en tiempo } \\
\text { real con los participantes del grupo (chat, vía } \\
\text { telefónica) }\end{array}$ & $88.5 \%$ & $11.5 \%$ & $0.0 \%$ \\
\hline $\begin{array}{l}\text { Los foros de trabajo presentan las herramientas } \\
\text { necesarias para desarrollar un buen trabajo } \\
\text { colaborativo. }\end{array}$ & $78.0 \%$ & $20.0 \%$ & $2.0 \%$ \\
\hline $\begin{array}{l}\text { El docente favoreció el trabajo colaborativo con } \\
\text { aportes adecuados. }\end{array}$ & $87.5 \%$ & $12.5 \%$ & $0.0 \%$ \\
\hline $\begin{array}{l}\text { El docente resolvió adecuadamente las dudas } \\
\text { presentadas por los estudiantes. }\end{array}$ & $85.5 \%$ & $14.5 \%$ & $0.0 \%$ \\
\hline $\begin{array}{l}\text { La función del docente como motivador en el } \\
\text { trabajo colaborativo, fue importante. }\end{array}$ & $89.0 \%$ & $11.0 \%$ & $0.0 \%$ \\
\hline $\begin{array}{l}\text { La evaluación del trabajo colaborativo se realizó } \\
\text { siguiendo la rúbrica. }\end{array}$ & $80.0 \%$ & $18.5 \%$ & $1.5 \%$ \\
\hline $\begin{array}{l}\text { La participación individual dentro del foro de } \\
\text { trabajo fue bien evaluada. }\end{array}$ & $88.5 \%$ & $9.0 \%$ & $2.5 \%$ \\
\hline $\begin{array}{l}\text { Se tuvo en cuenta la calidad de los aportes } \\
\text { presentados en el foro. }\end{array}$ & $86.0 \%$ & $13.5 \%$ & $0.5 \%$ \\
\hline $\begin{array}{l}\text { Las rúbricas de evaluación detallaban } \\
\text { claramente cada uno de los ítems a evaluar de } \\
\text { acuerdo a la guía de trabajo. }\end{array}$ & $83.5 \%$ & 16.05 & $0.5 \%$ \\
\hline \multicolumn{1}{c}{ Promedio general } & $\mathbf{8 3 . 4 \%}$ & $\mathbf{1 4 . 2 \%}$ & $\mathbf{2 . 4 \%}$ \\
\hline
\end{tabular}

Para finalizar, se pretende verificar si existen diferencias entre las puntaciones dadas por los estudiantes encuestados según el programa académico que actualmente están cursando. Para ello, se realizó la sumatoria de los veintitrés ítems asociados con la escala Likert, luego esta sumatoria oscila en el rango de 1 a 115 puntos, con calificación media de 69 puntos. Por medio de la gráfica 2 se puede verificar que debido a que los cuatro diagramas se solapan, se puede concluir que no existen diferencias significativas en las opiniones de los estudiantes respecto a las actividades de aprendizaje colaborativo desarrollado al interior de cada programa de pregrado. De la misma gráfica se observa que en todos los casos el promedio es muy superior a la media de la escala lo que evidencia el nivel de favorabilidad respecto a los ítems enunciados. En este sentido, los estudiantes de Licenciatura en Educación Infantil tienen opiniones muy afines (diagrama más corto), en contraste con los estudiantes de Licenciatura en Matemáticas cuyas opiniones son más diversas y se ve reflejado en el largo de la caja y con la calificación mediana más baja de todos los grupos. 


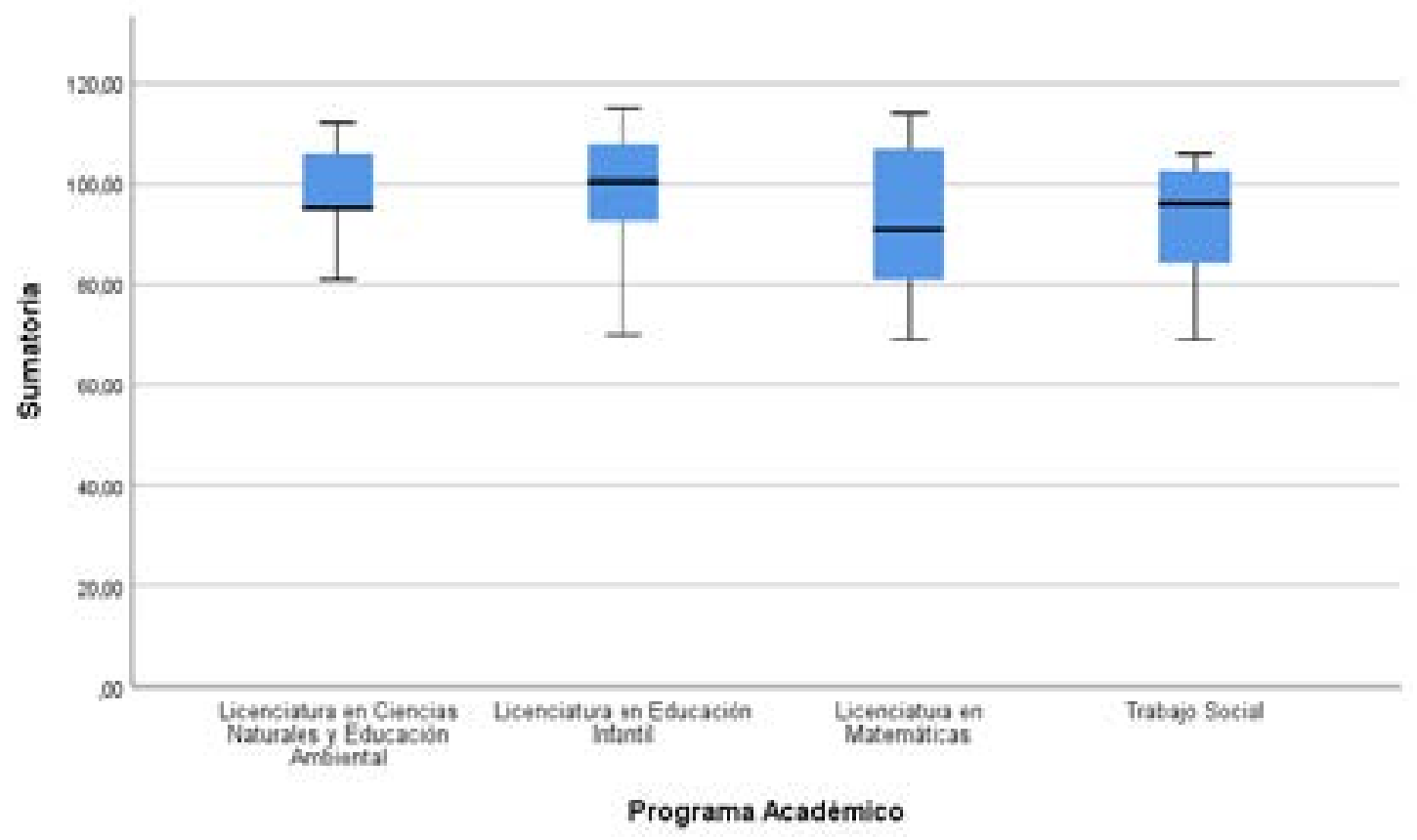

Gráfico 2. Diagrama de cajas y bigotes respecto al programa académico que cursa el estudiante

\section{CONCLUSIONES Y DISCUSIÓN}

El estudio logró develar elementos importantes en cuanto a la concepción positiva que tienen los estudiantes sobre el aprendizaje colaborativo en contextos no presenciales. Se puede concluir que las actividades realizadas en el periodo de tiempo analizado -segundo semestre de 2021-potenciaron de manera significativa el trabajo colaborativo en los estudiantes. En este contexto, es importante citar estudios como los de Gutiérrez y Gallego (2017), Prada, Gamboa y Avendaño (2021) quienes afirman que el aprendizaje colaborativo en escenarios de virtualidad genera un espacio importante de cohesión grupal, así mismo se presenta una comunicación más abierta y afectiva, siempre y cuando sea guiada de manera adecuada por los profesores.

El papel de los profesores es fundamental para detonar el aprendizaje en espacios de trabajo colaborativo. En este sentido, los resultados revelan que los docentes logran proveer a los alumnos de guías y orientaciones con objetivos y metas claras, con instrucciones precisas, con actividades pertinentes y tiempos coherentes. Se evidencia entonces según estas concepciones que uno de los elementos fundamentales para que el aprendizaje colaborativo se desarrolle de manera eficaz, es la buena gestión escolar del maestro. Con respecto a esta evidencia, es necesario señalar que uno de los elementos fundamentales para que el maestro pueda construir ambientes virtuales significativos y que promuevan el aprendizaje colaborativo es la innovación educativa en la educación no tradicional apoyada por tecnologías digitales (Sabulsky \& Ayelén, 2017; Lupión y Martín, 2016; Vargas, Gamboa y Prada, 2021; Rosas, 2006).

Dentro de los resultados más relevantes del estudio se destaca lo que podemos llamar un asomo a la alteridad virtual (Mesa y Leal, 2014; Moncada y Sánchez, 2017; Bohórquez y Muñoz, 2021). donde los estudiantes se apropian de las tecnologías digitales y de manera inconsciente 
se acercan a sus compañeros a pesar de la no presencialidad, generando espacios en donde se organizan roles, se definían tareas, se asumían responsabilidades y todos aportan a un objetivo común desde su propio espacio y desde el espacio del otro.

\section{REFERENCIAS BIBLIOGRAFÍCAS}

Bohórquez Martínez, N. D., \& Muñoz Romero, M. C. (2021). La alteridad como problema en el proceso educativo: reflexiones desde el confinamiento (Doctoral dissertation, Corporación Universitaria Minuto de Dios).

Calzadilla, M. E. (2002). Aprendizaje colaborativo y tecnologías de la información y la comunicación. Revista lberoamericana De Educación, 29(1), 1-10. https://doi. org/10.35362/rie2912868

Celina Oviedo, H., \& Campo Arias, A. (2005). Aproximación al uso del coeficiente alfa de Cronbach. Revista colombiana de psiquiatría, 34(4), 572-580.

Coll, C. (2014). El Sentido del Aprendizaje Hoy: un Reto para la Innovación Educativa. Aula de Innovación Educativa, 232, 12 -17. http://hdl.handle.net/2445/65763

González, C. (2006). Tutorización, evaluación y aprendizaje colaborativo en el aula virtual: un enfoque práctico. Moodle Moot 2006. https://www.researchgate. net/profile/Carina-Gonzalez-Gonzalez/ publication/258222833_Tutorizacion_ evaluacion_y_aprendizaje_ colaborativo_en_el_aula virtual_un_enfoque_practicol links/00b495276a28ab1e3b000000/ Tutorizacion-evaluacion-y-aprendizajecolaborativo-en-el-aula-virtual-unenfoque-practico.pdf
Gutiérrez-Santiuste, E., \& Gallego-Arrufat, M. J. (2017). presencia social en un ambiente colaborativo virtual de aprendizaje: Análisis de una comunidad orientada a la indagación. Revista Mexicana de Investigación Educativa, 22(75), 1169-1186. https://webebsco.unicartagenaproxy. elogim.com/ehost/pdfviewer/ pdfviewer?vid $=5 \&$ sid $=$ b8a50d33$8805-4$ d 56 - a a 36 b1a4f436b665\%40sessionmgr4006

Hernández, N., González, M., Muñoz, P. (2014). La planificación del aprendizaje colaborativo en entornos virtuales., Revista Científica de Educomunicación Comunicar, 42, (XXI) 25 - 33. http:// dx.doi.org/10.3916/C42-2014-02

Hernández Suárez, C. A., Arévalo Duarte, M. A., \& Gamboa Suárez, A. A. (2016). Competencias TIC para el desarrollo profesional docente en educación básica. Praxis \& Saber, 7(14), 41-69.

Lupión Cobos, T., \& Martín Gámez, C. (2016). Desarrollo profesional docente de profesorado de secundaria en una experiencia de innovación mediante investigaciones escolares. Revista Eureka Sobre Enseñanza y Divulgación de Las Ciencias, 13(3), 686-704. https:// doi.org/10.25267/Rev_Eureka_ensen_ divulg_cienc.2016.v13.i3.13

Mesa, J. E. R., \& Leal, L. A. (2014). De la educación virtual a la virtualización de procesos educativos; una transición en el contexto de las formas emergentes de cibercultura. Análisis. Revista Colombiana de Humanidades, 46(84), 125-142.https://www.redalyc.org/ articulo.oa?id=515551535007 
Moncada, C., \& Sánchez, M. (2017). Didáctica digital: aproximaciones para una apuesta pedagógica desde la alteridad virtual. Bogotá, Colombia: Editorial Universidad Manuela Beltrán

Nodarse, F. A., Añorga, R. G., Hernández, E. S., de Armas Vázquez, Y., \& Montenegro, S. L. (1999). Un entorno para aprendizaje colaborativo: Sistemas hipermedia y aula virtual. IE Comunicaciones: Revista Iberoamericana de Informática Educativa, 2 (14), 14 20. https://dialnet.unirioja.es/servlet/ articulo?codigo $=4794506$

Prada Núñez, Raúl, \& Hernández Suárez, César Augusto y Gamboa, Audin Aloiso (2019). Usos y efectos de la implementación de una plataforma digital en el proceso de enseñanza de futuros docentes en matemáticas. Revista Virtual Universidad Católica del Norte, (57), 137-156. https://www.redalyc.org/ articulo.oa?id=194260035010

Prada, R., Gamboa, A., \& Avendaño, W. R. (2021). Apropiación de competencias comunicativas y tecnológicas en maestros en formación de un programa acreditado de alta calidad. Boletín Redipe, 10(7), 132-143. https://doi. org/10.36260/rbr.v10i7.1354

Rubia, B., \& Guitert, M. (2014). ¿La revolución de la enseñanza? El aprendizaje colaborativo en entornos virtuales (CSCL). Comunicar, XXI (42),1014. https://www.redalyc.org/articulo. oa? id=15830197002

Rosas Chávez, P. (2006). Gestar y gestionar la virtualidad: un análisis desde la práctica y las instituciones. Apertura: Revista de Innovación Educativa, 6(3), 24-35.
Sabulsky, G., \& Ayelén Arevalo, E. (2017). Profesores memorables, entre la vocación y la práctica hay lugar para las TIC. Cuadernos de Educación, 15(15), 258-272.

Vargas Minorta L, Gamboa Suárez AA, Prada Núñez R. (2021). Pedagogía por proyectos y secuencia didáctica: una alternativa para el desarrollo de competencias comunicativas. Revista Boletín Redipe, 10(10), 115-124. https:// doi.org/10.36260/rbr.v10i10.1470 